\title{
Ocorrência natural de Beauveria bassiana (Bals.) Vuill. (Moniliales: Moniliaceae) e patogenicidade sobre Protortonia navesi Fonseca (Hemiptera: Monophlebiidae) na cultura da mandioca, em Marechal Cândido Rondon, Paraná
}

\author{
Natural occurrence of Beauveria bassiana (Bals.) Vuill. (Moniliales: \\ Moniliaceae) and pathogenicity on Protortonia navesi Fonseca (Hemiptera: \\ Monophlebiidae) in cassava plantation, in Marechal Cândido Rondon, Paraná
}

\author{
Tânia Mari Vicentini Prestes ${ }^{1 *}$, Vanda Pietrowski ${ }^{2}$, Agostinho Zanini ${ }^{1}$, Marina Andressa Formentini ${ }^{3}$, \\ Luis Francisco Angeli Alves ${ }^{3}$
}

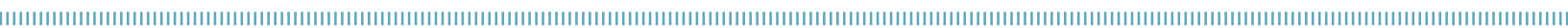

RESUMO: O objetivo deste estudo foi registrar a primeira ocorrência natural e patogenicidade da associaçáo entre Beauveria bassiana e Protortonia navesi na cultura da mandioca. Para isso, ninfas e adultos de $P$. navesi infectados foram coletados em amostragens realizadas quinzenalmente, na área do Instituto Agronômico do Paraná, em Marechal Cândido Rondon, de janeiro a dezembro de 2012. Amostras foram analisadas e, utilizando-se meio de cultura seletivo de aveia, foi isolado o fungo a partir dos cadáveres, sendo identificado como Beauveria bassiana (Bals.) Vuill., armazenado na Coleção de Fungos Entomopatogênicos do Laboratório de Biotecnologia Agrícola, sob o código Unioeste 78. Para comprovar sua patogenicidade, foi adotado o postulado de Koch, aplicando-se o fungo $\left(10^{9}\right.$ conídios $\left./ \mathrm{mL}\right)$ sobre ninfas e adultos de $P$. navesi obtidos da criação de laboratório. Após 10 dias de incubação, verificou-se $18 \%$ de mortalidade confirmada, sendo considerada baixa a atividade sobre a cochonilha $P$. navesi.

PALAVRAS-CHAVE: controle biológico; fungo entomopatogênico; cochonilha.

\begin{abstract}
The objective of this study was to record the first natural occurrence and pathogenicity of the association between Beauveria bassiana and Protortonia navesi in the cassava crop. Infected $P$. navesi nymphs and adults were collected in samples fortnightly in the Agronomic Institute of Paraná, in Marechal Cândido Rondon, from January to December 2012. Samples were analyzed at the Laboratory of Agricultural Biotechnology. The entomopathogenic fungus was isolated from corpses using selective oatmeal culture medium, and multiplied for sporulation being identified as Beauveria bassiana (Bals.) Vuill. The fungus was stored in the Collection of Entomopathogenic Fungi Laboratory of Agricultural Biotechnology, identified as Unioeste 78. To prove the pathogenicity of the found isolate, bioassays were performed following the postulate of Koch, the fungi suspension was sprayed $\left(10^{\circ}\right.$ conídios $\left.\mathrm{mL}\right)$, on nymphs and adults of $P$. navesi obtained from laboratory rearing. After 10 days of incubation there was $18 \%$ mortality confirmed, being considered low actgivity on mealybugs Protortonia.
\end{abstract}

KEYWORDS: biological control; entomopathogenic fungus; mealybugs. 
Protortonia navesi Fonseca (Hemiptera: Monophlebiidae) é uma cochonilha nativa do Brasil, com ocorrência registrada na cultura da mandioca e nas plantas daninhas no Distrito Federal e na regiâo oeste do estado do Paraná (Oliveira et al., 2008; Pietrowski et al., 2010).

A espécie foi descrita com potencial de danos elevados na cultura da mandioca, principalmente pela redução do poder germinativo das manivas em torno de $30 \%$, reduçấo do acúmulo de reserva, diminuição da estatura das plantas, redução da área foliar e do peso das raízes, em média, de 70\%, causando morte de 80\% das plantas (Oliveira; Fialho, 2005).

A disseminação da cochonilha ocorre principalmente pelas manivas infestadas, no transporte de máquinas e implementos, vestuário de pessoas que circulam em áreas infestadas e pelas formigas doceiras, as quais se encontram associadas (Oliveira et al., 2005).

Devido ao hábito críptico de desenvolvimento das cochonilhas, os métodos convencionais de controle são ineficazes, sendo mais utilizadas as práticas de rotaçáo de culturas, controle biológico, plantas sadias e eliminação de hospedeiros alternativos (Hajı et al., 2004), náo sendo recomendado o uso de inseticidas de contato ou sistêmico na área foliar, pois tais produtos náo apresentam bons resultados, devido à reinfestação pelas cochonilhas que estão nas raízes (Oliveira et al., 2005).

O controle biológico com o fungo Beauveria bassiana (Bals.) Vuill. é considerado eficiente no controle de diversas pragas (Alves, 1998). No entanto, para a cochonilha $P$. navesi, não foram encontrados estudos com esse entomopatógeno, havendo, contudo, trabalhos com outras cochonilhas, como a pesquisa com a raiz do cafeeiro, Dysmicoccus texensis (Tinsley) (Hemiptera, Pseudococcidae), realizada por Andaló et al. (2004), os quais verificaram que os isolados de B. bassiana (UEL 114) causaram mortalidade (62\%) em relação às demais espécies testadas. Também para a cochonilha do abacaxizeiro, D. brevipes, para a qual foi confirmado que $B$. bassiana mostrou-se com potencial para utlização no controle, pois em laboratório causou mortalidade de 53\% (SANTOS et al., 2013).

Foi verificada a viabilidade do uso de B. bassiana no controle da cochonilha ortezia (Praelongorthezia praelonga) (Douglas, 1891) (Hemiptera, Orthziidae), praga-chave dos citros, apresentando resultados promissores (BENVENGA et al., 2011). Ainda na mesma cultura esse entomopatógeno é citado para o controle de ninfas de $2^{\circ}$ ínstar de Planacoccus citri (Risso, 1803) (Hemiptera, Pseudococcidae) em laboratório (MasCarin et al., 2011).

$\mathrm{Na}$ cultura da mandioca, em estudo com a cochonilha Phenacoccus manihoti (Matile-Ferrero) (Hemiptera, Pseudococcidae), verificaram-se baixa atividade de B. bassiana sobre ninfas dessa cochonilha (BARILLI et al., 2011).

Assim, diante dos poucos métodos de controle disponíveis e da escassez de trabalhos científicos para essa praga, este estudo teve como objetivo registrar a primeira associação natural entre $B$. bassiana e $P$. navesi na cultura da mandioca e verificar a patogenicidade do fungo ao inseto.

Durante a realizaçáo do estudo de bioecologia na área do Instituto Agronômico do Paraná (IAPAR), localizada no distrito de Porto Mendes, Marechal Cândido Rondon (PR) ( $24^{\circ} 30^{\prime} 32,36^{\prime \prime}$ S, 54 18' 43,87'O, altitude $241 \mathrm{~m}$ ), foram coletados cadáveres de cochonilhas com sinais do fungo. Esses cadáveres foram individualizados em frascos de vidro com tampa e avaliados no Laboratório de Biotecnologia Agrícola da Universidade Estadual do Oeste do Paraná (UNIOESTE) de Cascavel.

O fungo entomopatogênico foi isolado dos cadáveres, utilizando-se o meio de cultura seletivo de aveia-Dodine (g/1.000 mL de água destilada): 20 ágar; 5,0 extrato de levedura; 4,6 mistura de sais; 10 glicose (Alves et al., 1998), incubado em $26 \pm 1^{\circ} \mathrm{C}$ e 12 horas de fotofase, durante 7 dias. Após, foi feita a raspagem dos conídios, sendo acondicionados em microtubos tipo Eppendorf e mantidos a $-10^{\circ} \mathrm{C}$. A multiplicação dos conídios foi feita em meio para esporulação (Alves, 1998). Inocularam-se os conídios nas placas que foram incubadas a $25 \pm 1^{\circ} \mathrm{C}$ e fotofase de 12 horas, por um período de 8 a 10 dias, para a obtenção da esporulaçấo do fungo. Coletaram-se amostras do fungo, raspando-se a superfície dos meios de cultura e lâmina de vidro contendo Amann lactofenol com cotton blue (0,5\%), observou-se ao microscópio de luz (400x). O fungo foi identificado como B. bassiana com base nos conídios globosos e fiálides com a parte basal dilatada terminando em zigue-zague (Alves, 1998; Humber, 2012).

Armazenou-se o isolado em tubos de vidro fechados com filme de PVC, em refrigeração $\left(-10^{\circ} \mathrm{C}\right)$, cadastrado na Coleção de Fungos Entomopatogênicos do Laboratório de Biotecnologia Agrícola da UNIOESTE (CFEUnioeste) (http://splink.cria. org.br/manager/detail?setlang=pt\&resource=CFEUnioeste), denominado Unioeste 78.

A fim de comprovar a patogenicidade do isolado encontrado, foram realizados bioensaios seguindo o postulado de Koch (Alves et al., 1998). Para isso, o isolado foi novamente multiplicado conforme descrito anteriormente. Após esse período, os conídios foram coletados, raspando-se a superfície do meio de cultura, transferindo-os para tubos de vidro esterilizados acrescidos de soluçáo de água destilada contendo Tween $^{\mathbb{B}^{\circ}} 80(0,01 \%)$. A suspensáo foi padronizada na concentração de $10^{9}$ conídios $/ \mathrm{mL}$, com o auxílio de uma câmara de Neubauer.

Cochonilhas de $P$. navesi de terceiro ínstar e adultas provenientes da criação do laboratório foram coletadas e transferidas, com o auxílio de pincel umedecido, para segmentos de raízes de mandioca, com oito meses de desenvolvimento, previamente escovados, lavados com água e hipoclorito de sódio a $1 \%$, revestidos com parafina líquida e acondicionados em caixas do tipo gerbox de acrílico. Logo após, $1 \mathrm{~mL}$ da suspensão fúngica foi pulverizado, utilizando-se Torre de 
Potter $\left(10 \mathrm{kgf} / \mathrm{cm}^{2}\right)$. Na testemunha, pulverizou-se água destilada + Tween $80(0,01 \%)$. Após a pulverização, as caixas de gerbox foram mantidas em sala climatizada $\left(26 \pm 1^{\circ} \mathrm{C}\right.$, U.R. $60 \pm 10 \%$, no escuro). Diariamente, durante 10 dias, foram realizadas as avaliaçôes, retirando-se os cadáveres que foram desinfestados por imersão em solução alcoólica $70 \%$ e água destilada e mantidos em câmara úmida em $26 \pm 1^{\circ} \mathrm{C}$ e 12 horas de fotofase, para confirmaçáo da mortalidade pelo fungo. $\mathrm{O}$ experimento foi realizado segundo delineamento inteiramente casualizado com 3 repetiçóes, sendo cada repetição uma caixa gerbox contendo segmentos de raízes de mandioca com 15 cochonilhas.

Em todas as amostragens de campo foram encontradas cochonilhas infectadas pelo fungo $B$. bassiana, registrando-se maior número nos meses de março a junho, quando as médias de precipitação e temperaturas mínimas e máximas do período foram de $130 \mathrm{~mm}, 16$ e $27^{\circ} \mathrm{C}$, respectivamente, proporcionando, com isso, a manutenção da umidade no solo (Fig. 1). A temperatura influencia a germinação dos conídios de $B$. bassiana, sendo determinada a temperatura de $26^{\circ} \mathrm{C}$ como limite para a germinação, pois a de $32^{\circ} \mathrm{C}$ é totalmente inibida (Alexandre et al., 2006).

A mortalidade média confirmada do isolado Unioeste 78 foi de $18 \pm 16,2$. Resultados semelhantes de baixa patogenicidade (20\%) foram obtidos para P. citri com isolados de B. bassiana (ESALQ - 447) na cultura de citros (MASCARIN et al., 2011). Entretanto, resultados superiores de patogenicidade foram obtidos por ANDALó et al. (2004), que verificaram

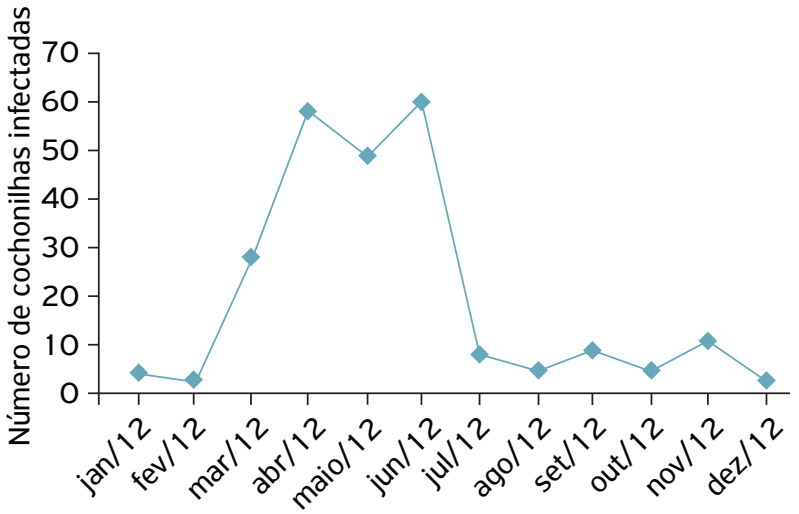

Figura 1. Número de cochonilhas infectadas, no período de janeiro a dezembro de 2012 , na cultura da mandioca da variedade fécula branca, em Porto Mendes, Marechal Cândido Rondon, Paraná

mortalidade para $D$. texensis de 50 a $65 \%$ para isolados de B. bassiana. Na cultura do abacaxizeiro, com a cochonilha D. brevipes, o mesmo fungo causou mortalidade de $53,2 \%$ (SANTOS et al., 2013).

Este é o primeiro estudo que registra a ocorrência de $B$. bassiana infectando $P$. navesi em raízes de mandioca. Contudo, a patogenicidade do isolado testado tenha sido baixa sobre a cochonilha. Sugere-se que outros estudos sejam realizados com a utilizaçấo de diferentes isolados de B. bassiana para o controle dessa espécie.

\section{REFERÊNCIAS}

ALEXANDRE, T.M.; ALVES, L.F.A.; NEVES, P.M.O.J.; ALVES, S.B. Efeito da temperatura e cama do aviário na virulência de Beauveria bassiana (Bals.) Vuill. E Metarhizium anisopliae (Metsch.) para o controle do cascudinho (Alphitobius diaperinus) (Panzer) (Coleoptera: Tenebrionidae). Neotropical Entomology, v.35, n. 1, p.75-82, 2006.

ALVES, S.B. Fungos entomopatogênicos. In: ALVES, S.B. (ed.). Controle microbiano de insetos. Piracicaba: FEALQ, 1998. p.289-381.

ALVES, S.B.; ALMEIDA, J.E.M.; MOINO, J.R.A.; ALVES, L.F.A. Técnicas de laboratório. In: ALVES, S.B. (ed.). Controle microbiano de insetos. Piracicaba: FEALQ, 1998. p.637-711.

ANDALÓ, V.; MOINO JÚNIOR, A.; SANTA-CECILIA, L.V.C.; SOUZA, G.C. Seleção de isolados de fungos e nematóides entomopatogênicos para a cochonilha-da-raiz-do-cafeeiro Dysmicoccus texensis (TINSLEY). Arquivos do Instituto Biológico, v.71, n.2, p.181-187, 2004.
BARILLI, D.R.; RHEINHEIMER, A.R.; MIRANDA, A.M.; MODOLON, T.A.; PIETROWSKI, V.; ALVES, F.A. Seleção de isolados de fungos entomopatogênicos no controle da mosca-branca (Bemisia tuberculata Matile-Ferrero) na cultura da mandioca. In: CONGRESSO BRASILEIRO DE AGROECOLOGIA, 6., 2011 , Fortaleza, CE. Cadernos de Agroecologia. Fortaleza, 2011 . v.6, n.2.

BENVENGA, S.R.; GRAVENA, S.; SILVA, J.L. da; JR. ARAUJO, N.; AMORIM, L.C.S. Manejo prático da cochonilha Ortézia em pomares de citrus. Citrus Research \& Technology, v.32, n. 1, p.39-52, 2011.

HAJI, F.N.P.; LIMA, M.P.L.; ALENCAR, J.A. de; BARBOSA, F.R.; FERREIRA, R.C.F.; MATTOS, M.A. de. Cochonilha-Pérola-da-Terra: Praga Emergente na Cultura da Uva, no Submédio do Vale do São Francisco. Petrolina: Embrapa, 2004. 8p. (Circular Técnica; n. 78).

HUMBER, R.A. Identificação de fungos entomopatogênicos. In: LACEY, L.A. Manual de Técnicas em Patologia de Invertebrados. Londres: Academic Press. 2012. p.151-187. 
MASCARIN, G.M.; PAULI, G.; LOPES, R.B. Suscetibilidade da cochonilha branca dos citros, Planacoccus citri, a Metarhizium anisopliae. Citrus Research \& Technology, v.32, n.3, p.155-160, 2011.

OLIVEIRA, C. M.; FIALHO, J. F. Disseminação e danos da cochonilha das raízes da mandioca Protortonia navesi Fonseca (Hemiptera: Margarodidae) em viveiro telado. In: CONGRESSO BRASILEIRO DE MANDIOCA, 1 1., 2005, Campo Grande. Anais. Campo Grande: Embrapa Agropecuária Oeste. CD-ROM.

OLIVEIRA, C.M.; FIALHO, J.F.; FONTES, J.R.A. Bioecologia, Disseminação e danos da cochonilha das raízes da mandioca Protortonia navesi FONSECA (HEMIPTERA: MARGARODIDAE). Planaltina: Embrapa - Cerrados, 2005. 29p.

OLIVEIRA, C.M.; FRIZZAS, M.R.; FIALHO, J.F.; GULLAN, P.J. Biology of Protortonia navesi (Hemiptera: Monophlebidae), a
New Cassava Pest in Brazil, with Notes on Its Behavior in the Field. Annals of the Entomological Society of America, v.101, p.779, 2008.

PIETROWSKI, V.; RINGENBERGER, R.; RHEINHEIMER, A.R.; BELLON, P.P.; GAZOLA, D.; MIRANDA, A.M. Insetos-praga da cultura da mandioca na região centro sul do Brasil. Marechal Cândido Rondon: UNIOESTE, 2010. 42p. (Documento Técnico).

SANTOS, L. H. dos.; SANCHES, N. S.; CARVALHO, R. S. Controle biológico da cochonilha do abacaxi Dysmicoccus brevipes pelo uso do fungo entomopatogênico Beauveria bassiana. In: JORNADA CIENTÍFICA EMBRAPA MANDIOCA E FRUTICULTURA, 7., 2013, Cruz das Almas. Anais. Cruz das Almas: Embrapa Mandioca e Fruticultura. Disponível em: <http://ainfo.cnptia.embrapa. br/digital/bitstream/item/89361/1/Controle-biologico-dacochonilha-054-13-LuizHenrique-Nilton.pdf>. 